\title{
Ethanol Induced EMG Changes and Muscle Force in a Patient with Polyneuropathy
}

\author{
MARKUS FÄRKKIL $\dddot{A}$ AND LEA LEINONEN \\ Department of Neurology and Neurophysiology University Hospital of Helsinki, \\ Helsinki Finland
}

Received for publication August 21, 1989

\begin{abstract}
Summary: A patient with chronic relapsing polyneuropathy noticed that when he took some alcohol in the evening his muscle force increased substantially during the next day. To measure this beneficial effect of ethanol we measured the force of hand grip, conduction velocities and distal latencies of ulnar and median nerves, and the power spectra of EMG during maximal and submaximal hand grip before and after alcohol ingestion. A $50 \%$ increase in the muscle force was documented 24 hours after the alcohol administration, with a concomitant increase of high frequency components in the power spectrum of EMG. Slight decreases in distal latencies were also measured.
\end{abstract}

Key words: ethanol - polyradiculitis - EMG - muscle force - nerve conduction velocity

\section{Introduction}

During a 2-year follow-up a 17-yearold school boy with chronic relapsing poly neuropathy was repeatedly treated with plasma exchanges. The plasma exchanges were started when the patient had difficulties in gait. He had observed that an increase of body temperature, e.g. after being in the Finnish sauna, had a deteriorating effect on his motor performance. He had also repeatedly experienced a substantial increse in the muscle force during the following day after drinking alcohol; the effect lasted from 4 to 8 hours. During the drunkenness he had noticed no change in the muscle force.

To document the effect of ethanol on neuromuscular system and to find out whether any concomitant changes could be detected with our routine neurophysiological means, we measured the force of hand grip, the power spectrum of EMG, and the impulse conduction in the median and ulnar nerves both before and after ethanol administration.

\section{Case report}

The symptoms began in November 1981 with a weakness in the legs observable when playing football. During the following weeks the weakness spread to the upper extremities; numbness was observed at the plantar sides of the feet. In January 1982 the patient was taken into the Department of Neurology; at this time he could not type and had difficulties in walking without support. Family history for neurological diseases was negative. In the clinical examination the prominent weakness of the extremities was symmetrical, tendon reflexes were absent, vibration sensitivity was decreased distally on the lower extremities. No orthostatic hypotension or changes in bowel function were observed; cranial nerves were intact. 


\section{Laboratory tests}

CSF showed one leukocyte, no red blood cells, a glucose level $3.4 \mathrm{mmol} /$ litre, and total protein of 1.2 grams per litre. CSF-IgG was risen, $153 \mathrm{mg}$ per litre, and $\mathrm{CSF}$ IgG/serum IgG ratio was normal. Bacterial and fungal cultures of CSF and viral and treponemal serology were negative. The following tests were normal or negative: complete blood count, erythrocyte sedimentation rate, serum protein electrophoresis, antinuclear antibody, rheumatoid factor, cold agglutinins, complement, acute phase proteins, creatine, glucose, transaminases, electrolytes, creatine kinase, urinanalysis, ECG, and chest $\mathrm{x}$-ray.

The electromyographies were first performed three months after the beginning of the symptoms. At that time fibrillation potentials were recorded in muscles, conduction velocities and distal latencies of peripheral nerves were slow (conduction of the order of $30 \mathrm{~m} / \mathrm{s}$ ), responses elicited by sensory stimulation were diminished or could not be recorded, and motor responses were small, long and polyphasic. During the diagnostic phase, the Tensilon $^{\mathrm{R}}$-test was done and was negative.

\section{Methods}

The force of hand grip was measured with a strain gauge dynamometer. The patient gripped the handle of two metal bars, and the grip force was documented on paper with ink-writer. He was first taught to use the apparatus so that the results were reproducible. During the measurements he was instructed to grip maximally twice for two seconds, with an interval of 15 seconds. The method and its reproducibility is presented in more detail in our earlier paper (Färkkilä et al. 1980).

The mean of these results was taken as an index of grip force. The interindividual variability for this patient in different measurements was small, only \pm 8 arbitrary units in the measurements done for him in the long course of his disease. Results described in this report were obtained with the right hand (the patient was right-handed). The variability of hand grip force was smallest, when measuring weakhanded persons.

Distal latencies (DL) and motor conduction velocities (MCV) were measured with bipolar surface electrodes from the ulnar and median nerves, sensory conduction velocities (SCV) were measured from the right radial nerve antidromically and from the median nerve orthodromically with bipolar surface electrodes. All stimuli were rectangular electric pulses of $0.5 \mathrm{~ms}$ duration. We first tried pulses of $0.2 \mathrm{~ms}$ with the maximal amplitude $(300 \mathrm{~V})$ obtainable from the equipment (Medelec) but the responses were of much lower amplitude. During the consecutive examinations (within two or three days) we used the same electrode locations which been marked on the skin with permanent 31 and $33^{\circ} \mathrm{C}$.

During the consequtive examinations the temperature was kept constant with a thermal radiator. The power spectra of EMG was measured with surface electrodes over the right palmaris longus during moderate and maximal voluntary grips of the dynamometer. EMG was sampled in epochs of $1.0 \mathrm{~s}$ and the power spectrum of the signal (frequency band $16-500 \mathrm{~Hz}$; $\mathrm{m}$ Fig. 1 shown, however, only up to 300 $\mathrm{Hz}$ ) was calculated with a computer. Several consequtive epochs were sampled to demonstrate the stability of the results. The mean frequency of the signal was calculated by computing the mobility parameter of the signal with normalized slope descriptors (Hjort, 1970). EMG activity was recorded from the resting right first dorsal interossus with a concentric needle electrode (Disa) before and after 


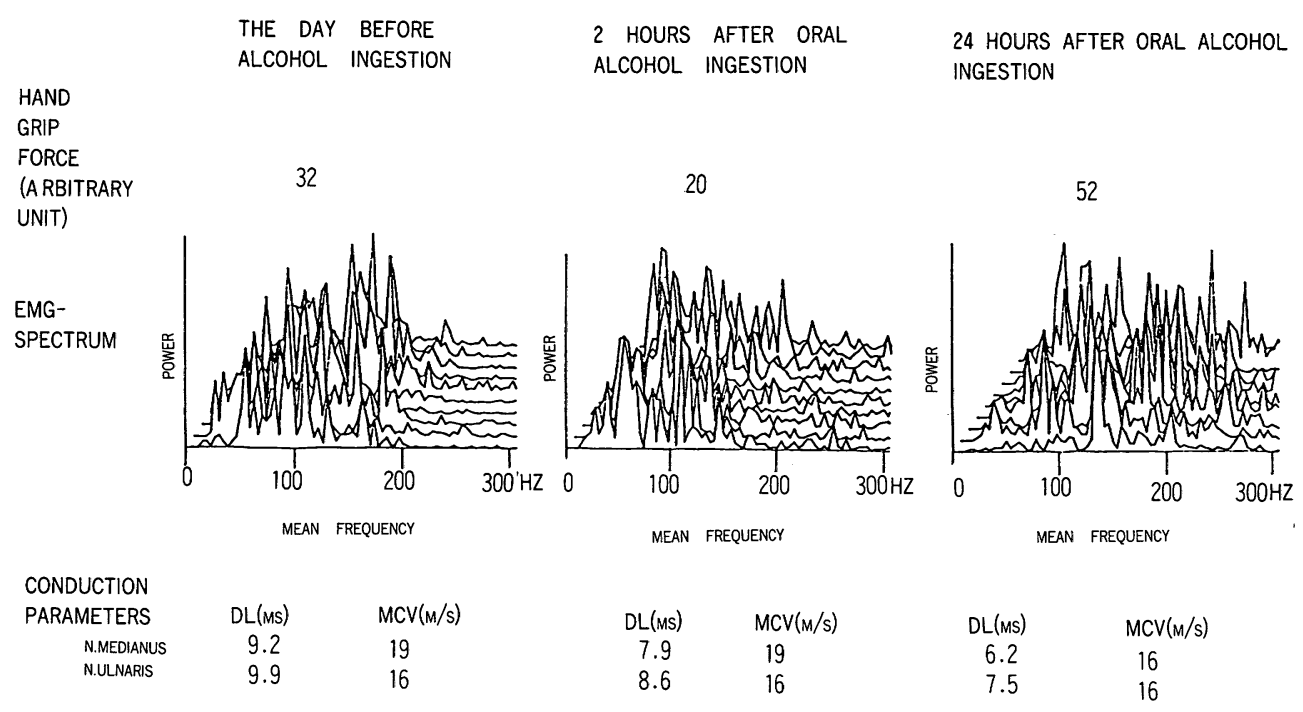

Fig. 1. Hand grip force measurement, EMG spectrum and nerve conduction parameters before alcohol ingestion, during the drunkness and during the hang over period.

the alcohol administration.

All measurements were made between 8 and $10 \mathrm{a} . \mathrm{m}$. on the day before the administration of ethanol, on the day of the administration and one day after. The distal latencies and conduction velocities were determined for 7 nerves on the day before the first plasma exchange and the day after plasma exchange. No pharmacological treatment was used at that time. The patient was given to drink $70 \mathrm{~g}$ absolute ethanol $(1 \mathrm{~g} / \mathrm{kg})$ in $600 \mathrm{ml}$ of orange juice between 6 and 7 a.m.

\section{Results and comments}

After the administration of ethanol the distal latencies decreased, Fig. 1. The grip force was slightly decreased immediately after the alcohol ingestion (perhaps lack of maximal effort) and increased thereafter so that after $24 \mathrm{~h}$ an increase of $60 \%$ was obseved, Fig. 1 . The EMGspectrum determined during maximal contraction showed a simultaneous increase in high frequency components, Fig. 1 ; The day following the alcohol ingestion abundant motor unit activity was recorded in the first dorsal interosseus with a needle electrode. The activity was identified as fasciculations and "bizarre repetitive potentials". The continuous motor unit activity could also be visually observed from the movements of the skin; it looked similar in the proximal parts of the upper extremities. Fasciculations were minimal before the ethanol administration. No fibrillations were recorded in the dorsal interosseus; probably because the study was carried out about one year after the onset of the illness.

There were no greater changes in the conduction velocities during the course of the follow-up, the measurement started 2.5 months after the first symptoms, Table 1. However, at the end of the follow-up the distal latencies had shortened and the sensory response of radial nerve recovered. This is perhaps due to re- 
TABLE 1

The EMG findings at different stages of the follow-up

\begin{tabular}{|c|c|c|c|c|}
\hline \multicolumn{2}{|c|}{$\begin{array}{l}\text { Conduction } \\
\text { Parameters }\end{array}$} & \multicolumn{3}{|c|}{ Duration of Symptoms } \\
\hline $\begin{array}{l}\mathrm{DL} \quad(\mathrm{m} / \mathrm{s}) \\
\mathrm{MCV}(\mathrm{m} / \mathrm{s}) \\
\mathrm{SCV}(\mathrm{m} / \mathrm{s})\end{array}$ & & 2.5 Months & 1 Years & 2 Years \\
\hline N. Medianus & $\begin{array}{l}\mathrm{DL} \\
\mathrm{MCV} \\
\mathrm{SCV}\end{array}$ & $\begin{array}{c}8.0 \\
25 \\
\text { No Response }\end{array}$ & $\begin{array}{c}9.2 \\
19 \\
\text { No Response }\end{array}$ & $\begin{array}{c}5.4 \\
17 \\
\text { No Response }\end{array}$ \\
\hline N. Ulnaris & $\begin{array}{l}\mathrm{DL} \\
\mathrm{MCV}\end{array}$ & $\begin{array}{l}14.8 \\
33\end{array}$ & $\begin{array}{l}9.9 \\
16\end{array}$ & $\begin{array}{l}6.2 \\
14\end{array}$ \\
\hline N. Radialis & SCV & No Response & 28 & 29 \\
\hline
\end{tabular}

peated plasma exchange treatment, which the patient received.

The study showed a substantial increase in muscle force after ethanol ingestion. The concomitant changes in EMG power spectrum were in agreement with this finding. The effect required several hours to develop, the exact time course was not monitored. Simultaneously with the increase in muscle force, a decrease of motor distal latencies was measured. Similar changes in conduction velocities have previously been reported after ethanol administration in a patient with polyradiculitis. (Juntunen and Sainio, 1976). The patient could repeat this effect during successive weekends, when drinking alcohol.

Ethanol causes a significant increase in the urinary excretion of catecholamines lasting at least $8 \mathrm{~h}$ after a single dose (Perman, 1958; Ylikahri et al. 1980). Ethanol also stimulates the excretion of cortisol, probably not via ACTH (Ylikahri et al. 1980). Plasma testosterone falls for $12 \mathrm{~h}$ after alcohol intake (Ylikahri and Huttunen, 1977). The secretion of prolactine increases rapidly and the response is abolished in $12 \mathrm{~h}$ (Wright, 1978). Ethanol may thus have its delayed effect on muscle force through the increase of circulating catecholamines or through various steroid hormones. Anyhow, the neuromuscular transmitter is acetylcholine, not a catecholamine.

Increase in muscle force can also be due to membrane effect of alcohol. During the hangover period the irritability of membranes increases, perhaps due to catecholamines, and facilitates the conduction block, and so increases temporarily muscle force.

Steroids have a beneficial effect on chronic relapsing polyneuropathy (Dalakas and Engel, 1981). Our patient had received steroids, but he had not noticed a similar increase in muscle force as during hangover periods. Both corticosteroids and catecholamines can affect the conduction block in chronic polyradiculitis. It is probable that the alcohol induced increase in the muscle force is mostly due to catecholamines.

\section{References}

Dalakas, M.C. and EnGel, W.K. (1981). Chronic relapsing (dysimmune) polyneuropathy: $\mathrm{Pa}$ thogenesis and treatment. Ann. Neurol. 9 (suppl), 134-145. 
Färkkilä, M. A., Pyykkö, V.I., Korhonen, O.S. and Starck, J. (1980). Vibration induced decrease in the muscle force in lumberjacks. Eur. J. Appl. Physiol. 43, 1-9.

HJoRth, B. (1970). EEG analysis based on time domain properties. Electroenceph. Clin. Neurophysiol. 29, 306-310.

Juntunen, J. and Sainio, K. (1976). Effects of alcohol upon symptoms of polyneuropathy. Lancet I, 254.

Perman, F.S. (1958). The effect of ethyl alcohol on the secretion from the adrenal medulla in man. Acta Physiol. Scand. 44, 241-247.

YlikAhri, R. H., Huttunen, M.O. and Härkönen, M. (1980). Hormonal changes during alcohol intoxication and withdrawal. Pharmacol. Biochem. Behav. 13 suppl 1, 131-137.

YlikAhri, R. H. and Huttunen, M.O. (1977). Metabolic and endocrine pathology during hangover. In Alcohol intoxication and withdrawal. ed. M. Gross. New York: Plenum Press.

Wright, J. (1978). Endocrine effects of alcohol. Clin. Endocrinol. Metab. 7, 351-367. 